\title{
The peculiar high-mass X-ray binary 1ES 1210-646
}

\author{
N. Masetti ${ }^{1}$, R. Landi $^{1}$, V. Sguera ${ }^{1}$, F. Capitanio ${ }^{2}$, L. Bassani ${ }^{1}$, A. Bazzano ${ }^{2}$, A. J. Bird ${ }^{3}$, A. Malizia ${ }^{1}$, and E. Palazzi ${ }^{1}$ \\ 1 INAF - Istituto di Astrofisica Spaziale e Fisica Cosmica di Bologna, via Gobetti 101, 40129 Bologna, Italy \\ e-mail: masetti@iasfbo.inaf.it \\ 2 INAF - Istituto di Astrofisica Spaziale e Fisica Cosmica di Roma, via Fosso del Cavaliere 100, 00133 Rome, Italy \\ 3 School of Physics \& Astronomy, University of Southampton, Southampton, Hampshire, SO17 1BJ, UK
}

Received 5 October 2009 / Accepted 7 December 2009

ABSTRACT

\begin{abstract}
Using data collected with the BeppoSAX, INTEGRAL and Swift satellites, we report and discuss the results of a study on the X-ray emission properties of the X-ray source 1ES 1210-646, recently classified as a high-mass X-ray binary through optical spectroscopy. This is the first in-depth analysis of the X-ray spectral characteristics of this source. We found that the flux of 1ES 1210-646 varies by a factor of $\sim 3$ on a timescale of hundreds of seconds and by a factor of at least 10 among observations acquired over a time span of several months. The X-ray spectrum of 1ES 1210-646 is described using a simple powerlaw shape or, in the case of INTEGRAL data, with a blackbody plus powerlaw model. Spectral variability is found in connection with different flux levels of the source. A strong and transient iron emission line with an energy of $\sim 6.7 \mathrm{keV}$ and an equivalent width of $\sim 1.6 \mathrm{keV}$ is detected when the source is found at an intermediate flux level. The line strength seems to be tied to the orbital motion of the accreting object, as this feature is only apparent at the periastron. Although the X-ray spectral description we find for the 1ES 1210-646 emission is quite atypical for a high-mass X-ray binary, the multiwavelegth information available for this object leads us to confirm this classification. The results presented here allow us instead to definitely rule out the possibility that 1ES 1210-646 is a (magnetic) cataclysmic variable as proposed previously and, in a broader sense, a white dwarf nature for the accretor is disfavoured. X-ray spectroscopic data actually suggest a neutron star with a low magnetic field as the accreting object in this system.
\end{abstract}

Key words. binaries: general - X-rays: binaries - stars: neutron - techniques: spectroscopic X-rays: individuals: 1ES 1210-646

\section{Introduction}

$\mathrm{X}$-ray binaries are interacting systems in which a compact object, neutron star (NS) or black hole $(\mathrm{BH})$ is accreting matter from a companion star. Depending on the mass of the latter, these systems are broadly divided into high-mass X-ray binaries (HMXBs; if the companion is a massive early type star) and low mass X-ray binaries (LMXBs; in the cases in which the secondary star is of late spectral type, in general with mass $\lesssim 1 M_{\odot}$ ).

Several X-ray binaries relatively bright in X-rays could not be properly identified and classified until recently due to the lack of a precise (arcsecond-sized) X-ray position: indeed, the X-ray characteristics may not provide enough elements for the correct classification of an X-ray binary as a HMXB or as a LMXB. This can therefore only be achieved through a multiwavelength approach, mostly with the synergic use of information acquired in X-ray and optical bands (although X-ray timing properties, such as bursts or pulsations, can give precise indications on the nature of the accretor). The subject of this paper, 1ES 1210-646, is indeed an object which fits the above description.

The X-ray source 1ES 1210-646 was first detected by the Uhuru satellite and reported (as 4U 1210-64) in the 4th Uhuru Catalogue (Forman et al. 1978) as a relatively bright and variable source with a 2-6 keV flux of $8.9 \times 10^{-11} \mathrm{erg} \mathrm{cm}^{-2} \mathrm{~s}^{-1}$, assuming

* Partly based on X-ray observations with INTEGRAL, an ESA project with instruments and science data centre funded by ESA member states (especially the PI countries: Denmark, France, Germany, Italy, Switzerland, Spain), Czech Republic and Poland, and with the participation of Russia and the USA. a Crab-like spectrum (below we will always assume this spectral shape for our X-ray flux estimates unless explicitly stated otherwise). It was also detected in the slew surveys performed with the Einstein (Elvis et al. 1992) and EXOSAT (Reynolds et al. 1999) satellites, at fluxes $1.7 \times 10^{-11} \mathrm{erg} \mathrm{cm}^{-2} \mathrm{~s}^{-1}(0.16-$ $3.5 \mathrm{keV})$ and $\sim 7 \times 10^{-10} \mathrm{erg} \mathrm{cm}^{-2} \mathrm{~s}^{-1}(1-8 \mathrm{keV})$, respectively. More recently, this source was detected by the wide-field cameras (WFCs; Jager et al. 1997) onboard BeppoSAX (Boella et al. 1997) at an average flux of $2.57 \times 10^{-10} \mathrm{erg} \mathrm{cm}^{-2} \mathrm{~s}^{-1}$ in the 2-10 keV (Verrecchia et al. 2007), and at hard X-rays above $20 \mathrm{keV}$ in the surveys (Bird et al. 2007; Krivonos et al. 2007) obtained with the IBIS instrument (Ubertini et al. 2003) onboard the INTEGRAL satellite (Winkler et al. 2003), at a flux of $1.1 \times 10^{-11} \mathrm{erg} \mathrm{cm}^{-2} \mathrm{~s}^{-1}$ in the $20-100 \mathrm{keV}$ band (Bird et al. 2007). High-energy emission from 1ES 1210-646 was also detected by Swift/BAT in the $14-195 \mathrm{keV}$ band with a flux $\sim 2 \times 10^{-11} \mathrm{erg} \mathrm{cm}^{-2} \mathrm{~s}^{-1}$ (Tueller et al. 2010; Cusumano et al. 2009).

Unfortunately, the above X-ray detections had positional errors of the order of several arcminutes or worse, which severely hampered the search for longer-wavelength counterparts and in turn the determination of the nature of this source.

Things changed thanks to a pointed observation performed with the X-ray telescope (XRT, Burrows et al. 2005) onboard the Swift satellite (Gehrels et al. 2004): according to Revnivtsev et al. (2007), these data revealed a clear counterpart of 1ES 1210-646 at coordinates $(\mathrm{J} 2000) \mathrm{RA}=12^{\mathrm{h}} 13^{\mathrm{m}} 14.702$, Dec $=$ $-64^{\circ} 52^{\prime} 30^{\prime} 89$, with a position uncertainty of $\sim 4^{\prime \prime}$. Revnivtsev et al. (2007) also stated that this source may be an intermediate 
Table 1. Log of the Swift/XRT observations used in this paper.

\begin{tabular}{ccccc}
\hline \hline $\begin{array}{c}\text { Obs. } \\
\text { number }\end{array}$ & Start day & $\begin{array}{c}\text { Start time } \\
(\mathrm{UT})\end{array}$ & $\begin{array}{c}\text { On-source } \\
\text { time }(\mathrm{ks})\end{array}$ & $\begin{array}{c}\text { Avg. 0.3-10 keV } \\
\text { countrate }\left(\mathrm{cts} \mathrm{s}^{-1}\right)\end{array}$ \\
\hline$\# 1$ & 10 Dec. 2006 & $17: 48: 48$ & 9.9 & $0.939 \pm 0.014$ \\
$\# 2$ & 07 Feb. 2008 & $15: 05: 24$ & 2.9 & $0.369 \pm 0.011$ \\
$\# 3$ & 24 Apr. 2008 & $07: 49: 26$ & 6.9 & $1.755 \pm 0.031$ \\
\hline
\end{tabular}

polar (i.e. magnetic) cataclysmic variable $(\mathrm{CV})$ on the basis of its $\mathrm{X}$-ray spectral appearance, in particular because of the presence of a strong iron emission line at $\sim 6.7 \mathrm{keV}$.

To firmly identify the nature of this source, Masetti et al. (2009) included the single optical object consistent with the XRT position of 1ES 1210-646 in their spectroscopic follow-up of INTEGRAL sources: from its spectrum they concluded that it is more likely a HMXB rather than a CV. This was corroborated by the discovery of a periodicity of 6.7 d (Corbet \& Mukai 2008) from the analysis of the long-term X-ray light curve of the source as seen by the All-Sky Monitor (ASM) onboard the RXTE satellite: if this modulation is interpreted as the orbital period of 1ES 1210-646, as Corbet \& Mukai (2008) did, it would strengthen the HMXB nature for this source. These authors also reported that the 2.5-20 keV X-ray spectrum of the source acquired close to the orbital maximum is typical of a HMXB; they also confirmed the presence of the iron emission line.

Despite the number of multiwavelength information gathered in the past few years on 1ES 1210-646, a deeper study of this source in X-rays is still lacking. In order to fill this gap and better constrain and define the observational X-ray properties of 1ES 1210-646, we collected and analysed the three available Swift/XRT observations of this source, along with the INTEGRAL/IBIS data from the 3rd IBIS survey (Bird et al. 2007) and the publicly available data collected with the two-unit coded-mask X-ray monitor JEM-X (Lund et al. 2003), also onboard INTEGRAL; we also performed a deeper analysis of the archival BeppoSAX/WFC data.

The paper is structured as follows. Section 2 will present the X-ray observations of 1ES 1210-646 considered in this work; in Sect. 3 the results of this multi-observatory X-ray campaign will be given, and in Sect. 4 a discussion on the source will be presented. Finally, in Sect. 5 we will outline the conclusions. Throughout the paper, if not indicated otherwise, uncertainties and limits are given at a $90 \%$ confidence level.

\section{Observations and data analysis}

\subsection{Swift: XRT data}

The field of 1ES 1210-646 was observed three times with XRT. All pointings were performed in photon counting mode (see Burrows et al. 2005, for details on this observing mode). The $\log$ of these observations is reported in Table 1.

The data reduction was performed using the XRTDAS v2.0.1 standard data pipeline package (xrtpipeline v0.10.6) to produce the final cleaned event files. As in observations \#1 and \#3 the XRT count rate of the source was high enough to produce data pile-up, we extracted the events in an annulus centred on the source and with an external radius of $57^{\prime \prime}$ and $68^{\prime \prime}$, respectively. The size of the inner circle was determined following the procedure described in Romano et al. (2006) and was $9^{\prime \prime}$ for observation 1 and $21^{\prime \prime}$ for observation 3 . In observation \#2 the pile-up was not an issue, so the data were extracted from a circle of a radius of $47^{\prime \prime}$. The source background was measured within a circular region with a radius of 95" located far from the source. The ancillary response file was generated with the task xrtmkarf (v0.5.2) within FTOOLS ${ }^{1}$ (Blackburn 1995), and accounts for both extraction region and PSF pile-up correction. We used the latest spectral redistribution matrices in the calibration database $^{2}$ (CALDB 2.3) maintained by HEASARC.

\subsection{INTEGRAL: IBIS and JEM-X data}

We extracted the spectral and time-series data of this source collected with ISGRI (Lebrun et al. 2003), which is the lowenergy coded-mask detector of the IBIS instrument onboard INTEGRAL. The ISGRI data set considered in this analysis consists of events in the 17-300 keV band coming from both fullycoded and partially-coded observations of the field of view of 1ES 1210-646. The time resolution for these data was that typical of the IBIS science windows (ScWs; $\sim 2 \mathrm{ks}$ ). Details on the whole procedure can be found in Bird et al. (2007). Hard X-ray long-term light curves and a time-averaged spectrum were then obtained from the available data and by the method described in Bird et al. (2006, 2007), for a total of $1168 \mathrm{ks}$ on-source collected in the time interval between October 2002 and April 2006.

Publicly-available 3-35 keV band JEM-X data of $1 E S$ 1210-646 were also collected. They were reduced and analysed as well with the OSA v7.0 software (for details about the JEM$X$ data analysis see Westergaard et al. 2003). We searched the entire JEM-X public data archive (from revolution 46 to 574) for pointings where 1ES 1210-646 was within the JEM-X fully coded field of view ( $\sim 2^{\circ} .4$ radius). As a result, a total of $70 \mathrm{ScWs}$ were selected, spanning the period from 28 May 2003 (Rev. 76) to 26 Jun. 2007 (Rev. 574).

It was found specifically that 1ES 1210-646 entered the fully-coded field of view of Unit 2 of JEM-X for $18 \mathrm{ks}$ between 28 May 2003 and 3 Jun. 2003, and for 116 ks in the one of Unit 1 between 20 Dec. 2004 and 26 Jun. 2007. In the first case, the source was not detected, whereas in the second instance it was in the 3-10 keV range; a more in-depth inspection of the latter JEM-X data set revealed that the source displayed two different states: a high flux state from Rev. 322 (2 Jun. 2005) to Rev. 324 (8 Jun. 2005) for a total exposure of $\sim 22 \mathrm{ks}$ (26- $\sigma$ detection) and a low flux state, fainter by a factor of $\sim 4$, in the remaining part of the data set, for a total exposure of $\sim 94 \mathrm{ks}(8-\sigma$ detection). The statistics were sufficient to extract a meaningful JEM$\mathrm{X}$ spectrum in the 3-10 keV band only during the high state of the source. Information on the source fluxes as detected with JEM-X are reported in Sect. 3.2.2.

\subsection{BeppoSAX: WFCs data}

The two WFCs on board BeppoSAX were sensitive in the $2-28 \mathrm{keV}$ range and were mounted $180^{\circ}$ apart and perpendicular to the pointing direction of the satellite, thus looking at two different sky zones during any pointing. In this way the WFCs secondary mode observations covered almost all of the sky with at least one of their serendipity pointings (typically with $100 \mathrm{ks}$ duration) during the six years of the BeppoSAX operational life.

We searched for 1ES 1210-64 detections in the archive ${ }^{3}$ of all available BeppoSAX WFC pointings. The source has been

\footnotetext{
1 Available at: http://heasarc.gsfc.nasa.gov/ftools/

2 Available at: http://heasarc.gsfc.nasa.gov/

docs/heasarc/caldb/caldb_intro.html

3 The WFCs data archive collected by the INAF-IASF of Rome (Italy) has been used for this analysis
} 
N. Masetti et al.: 1ES 1210-646, a peculiar high-mass X-ray binary

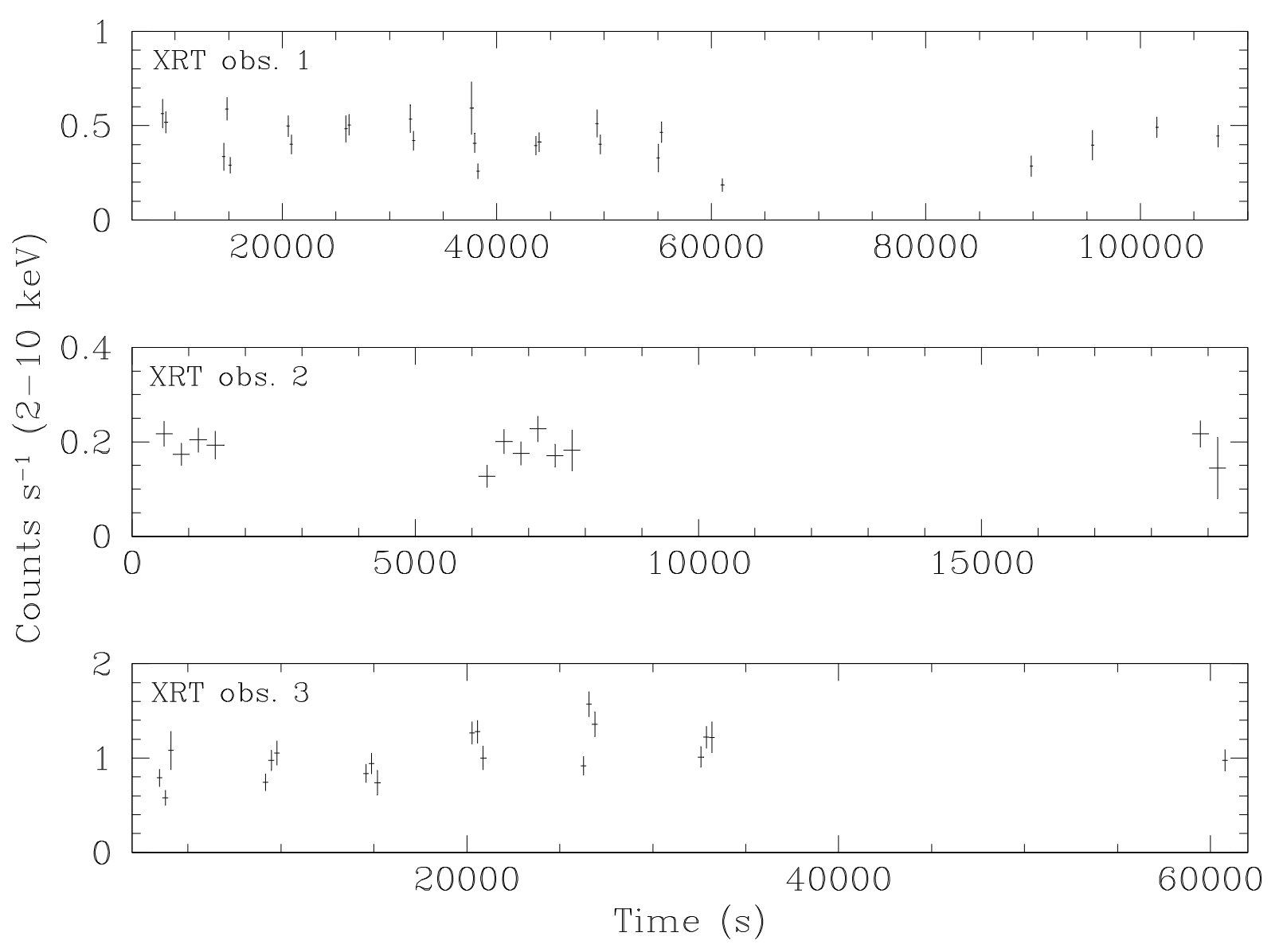

Fig. 1. Background-subtracted 2-10 keV X-ray light curves of 1ES 1210-646 as observed with XRT during observations \#1 (upper panel), \#2 (central panel) and \#3 (lower panel). All curves are rebinned at $300 \mathrm{~s}$. Times are expressed in seconds from the start time of each observation (see Table 1 for details). Marked variability on timescales of the order of hundreds of seconds can be seen, especially during observation \#1.

observed for a total of 1.5 Ms between April 1996 and April 2002, although it was detected in single pointing observations only between July 1996 and December 1997, in agreement with the highest source flux detections of the RXTE/ASM monitor ${ }^{4}$ (see also Corbet \& Mukai 2008).

Unfortunately, the WFCs were not sensitive enough in terms of spectral capability, and 1ES 1210-646 was too faint in each single pointing and also in the sum of the observations to permit the extraction of meaningful spectra. Thus, only information on the source flux could be extracted from the WFC data (see Sect. 3.2.3 for the results).

\section{Results}

\subsection{Light curves}

Figure 1 reports the 2-10 keV light curves of 1ES 1210-646 acquired during the three XRT pointings on this source. The data are background-subtracted and rebinned at $300 \mathrm{~s}$. Variability (up to a factor of $\sim 3$ in flux) of the order of hundreds of seconds can be noticed. Apart form this, one can see that the average 2$10 \mathrm{keV}$ source countrate varies notably among the observations, going from $\sim 0.15 \mathrm{cts} \mathrm{s}^{-1}$ of XRT pointing \#2 to $\sim 1 \mathrm{cts} \mathrm{s}^{-1}$ in XRT pointing \#3.

In order to see if these erratic behaviours implied spectral changes as a function of the source intensity within each XRT observation, we constructed an X-ray "colour-intensity diagram"

${ }^{4}$ http://xte.mit.edu/ASM_lc.html (see Fig. 2) using the total $0.3-10 \mathrm{keV}$ intensity and the hardness ratio between the $2-10 \mathrm{keV}$ and the $0.3-2 \mathrm{keV}$ count rates. In the figure, points from different observations are indicated with different symbols. From the diagram, it appears that the source gets slightly harder as its intensity rises, although it seems that within the same XRT observation 1ES 1210-646 shows a fairly constant hardness ratio: $\sim 1$ for XRT pointing \#1 and \#2, and $\sim 1.6$ for XRT pointing \#3.

Unfortunately, the wide gaps in the temporal coverage do not allow us to perform a meaningful timing analysis on any of the XRT pointings. For the same reason and because of sensitivity limits, no long-term periodicity analysis on the WFC, IBIS and JEM-X light curves of 1ES 1210-646 could reasonably be performed to confirm the modulation found by Corbet \& Mukai (2008). We note however that the source variability appears to be stronger at lower energies $(<20 \mathrm{keV})$, given that in the IBIS data it is continuously detected as a weak persistent object.

\subsection{Spectra}

In order to perform the spectral analysis on the X-ray data collected for 1ES 1210-646, the spectra from the detectors of all spacecraft but IBIS were rebinned to oversample by a factor of 3 the full width at half maximum of the energy resolution and to have a minimum of 20 counts per bin to reliably use the $\chi^{2}$ statistics; for IBIS, the rebinning was chosen to maximise the signal-to-noise ratio $(\mathrm{S} / \mathrm{N})$ for each bin. Data were then selected for all detectors when a sufficient number of counts were 


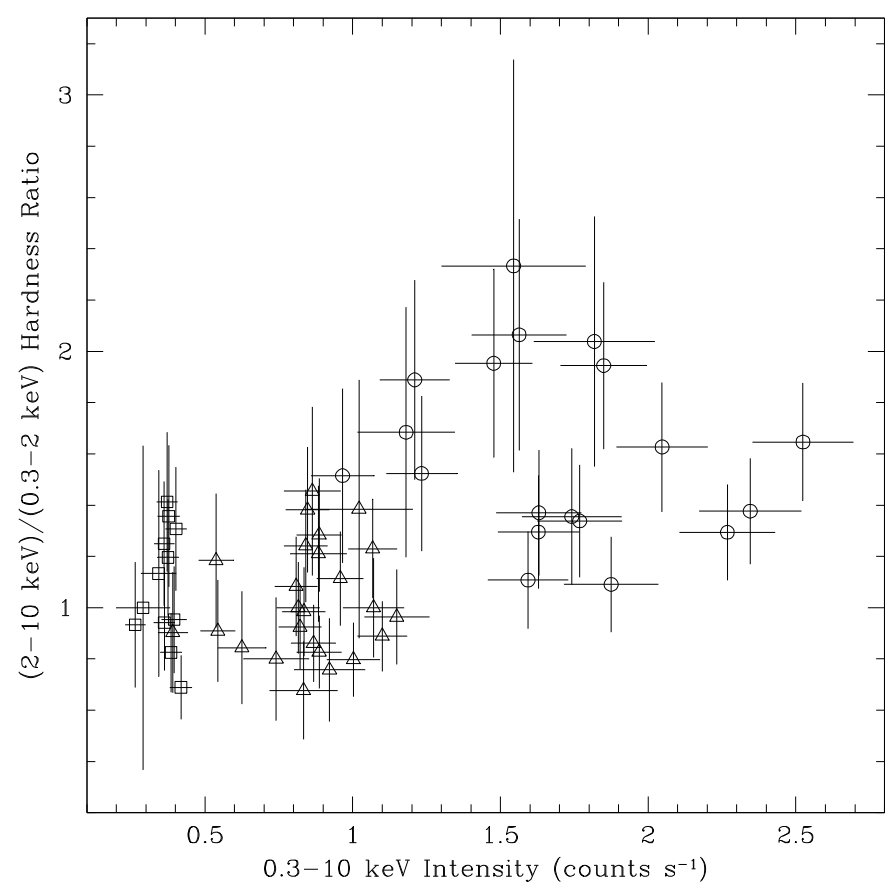

Fig. 2. X-ray colour-intensity diagram for 1ES 1210-646 constructed using the three XRT observations of the source. Points are obtained from the source X-ray light curves, subtracted of the background and rebinned at $300 \mathrm{~s}$. Triangles, squares and circles refer to XRT pointings \#1, \#2 and \#3, respectively. Error bars show 1- $\sigma$ confidence level uncertainties for each data point.

obtained in the energy ranges where the instrument responses are well determined. For the Swift/XRT observations, we considered the spectra averaged over each pointing given that no substantial variations in the spectral shape during each pointed observation were suggested by the inspection of the colour-intensity diagram of the source (see Sect. 3.1). For INTEGRAL the data were accumulated over the periods in which each instrument could secure spectra with acceptable $\mathrm{S} / \mathrm{N}$, that is, between 2 and 8 Jun. 2005 for JEM-X and the whole interval of the third survey for IBIS. Due to the tradeoff between spectral coverage and $\mathrm{S} / \mathrm{N}$, the spectral analysis was performed over the $0.3-9 \mathrm{keV}, 3-10 \mathrm{keV}$ and 20-100 bands for the XRT, JEM-X and IBIS data, respectively.

To analyse the X-ray spectra we used the package XSPEC (Dorman \& Arnaud 2001) v12.4.0ad. Several simple models like blackbody (BB), powerlaw, thermal bremsstrahlung, and hot diffuse gas emission (MEKAL in XSPEC; Mewe et al. 1985), were tested for the description of the spectral data. Spectral fitting which used more complex models like a powerlaw modified with a cutoff did not improve the results over those obtained with the aforementioned simpler desciptions because of the relatively low $\mathrm{S} / \mathrm{N}$ of the $\mathrm{X}$-ray spectra presented here.

To all models tested in this paper (the best-fit models for each satellite pointing are reported in Table 2) we applied a photoelectric absorption column, modelled with the cross sections of Morrison \& McCammon (1983; wabs in XSPEC notation) and with solar abundances as given by Anders \& Grevesse (1989), to describe the line-of-sight Galactic neutral hydrogen absorption towards 1ES 1210-646. For clarity, the luminosities listed in Table 2 refer to only one of the reported models (see Note in Table 2); it can however be said that they are basically independent of the considered best-fit model. Below, the acronym "d.o.f." means "degrees of freedom".

\subsubsection{XRT data}

The spectral analysis of the three XRT observations (see Table 2) indicates that the models which best describe the data are those with the powerlaw and the thermal bremsstrahlung, absorbed by a neutral hydrogen column with density $N_{\mathrm{H}} \sim 4$ $5 \times 10^{21}$ atoms $\mathrm{cm}^{-2}$. This value is comparable to the optical reddening along the line of sight as derived by Schlegel et al. (1998), once the empirical formula of Predehl \& Schmitt (1995) is applied; this indicates that the measured absorption is most likely of interstellar origin and none is locally present in 1ES 1210-646. The same conclusion was reached by Masetti et al. (2009) on the basis of the properties of the optical counterpart of this source.

Looking at Table 2 one also notes that with the powerlaw description the spectrum is relatively hard at the lowest X-ray fluxes (XRT pointing \#2), gets softer at an intermediate flux level (XRT pointing \#1) and eventually turns harder when the source is brightest (XRT pointing \#3); similarly the temperature steadily rises in the case of bremsstrahlung fits from $\sim 7.3$ to $\sim 22 \mathrm{keV}$ from XRT pointing \#1 to XRT pointing \#3.

The most fascinating spectral feature is the presence of a strong and variable iron emission line around $6.7 \mathrm{keV}$ which is evidently present only on XRT observation \#1 (as already noted by Revnivtsev et al. 2007) at an intermediate source flux and which is not detected at low and high flux levels (see Fig. 3). Assuming a Gaussian shape for the iron emission, the probability of a chance improvement of the fit over a simple powerlaw description in the spectrum of XRT pointing \#1 is $5.9 \times 10^{-10}$ according to the F-test distribution (Press et al. 1992). Although we are aware of the caveats and limitations of the F-test application in astrophysical context (e.g., Protassov et al. 2002), the above value for the chance improvement probability leaves little doubt that the detection of this emission line is quite robust. However, no emission is present in the X-ray spectra extracted from XRT observations \#2 and \#3 (see central and lower panels of Fig. 3).

Table 2 also presents the best-fit parameters describing this emission line. As one can see, they are largely independent of the model assumed for the description of the X-ray spectral continuum. Table 3 reports values or upper limits for the equivalent width (EW) of any iron emission line at $\sim 6.7 \mathrm{keV}$ in the spectrum of each pointed observation. As is evident, the line EW has a variability of at least a factor of 8 .

For the sake of completeness, we report that the BB emission does not provide a satisfactory description of the spectral data; the MEKAL model is not a viable option either, as it implies a variation of the plasma metal abundances of a factor of 100 among the three XRT observations due to the presence of the highly variable iron line described above. Likewise, any combination of two simple models (e.g., BB plus powerlaw) does not produce significant improvements of the spectral fitting in any of the three XRT pointings.

\subsubsection{JEM-X and IBIS data}

Next, we consider the combined JEM-X and IBIS spectra between 3 and $100 \mathrm{keV}$ (Fig. 4). We introduced a constant $\left(C_{\text {calib }}\right)$ to allow for intercalibration differences between the two instruments; this constant was left free to vary in the fits. We are aware that since the IBIS spectrum is accumulated over a much longer time span with respect to the JEM-X one (see Sect. 2.2), the flux variations seen from 1ES 1210-646 in particular below $10 \mathrm{keV}$ 
N. Masetti et al.: 1ES 1210-646, a peculiar high-mass X-ray binary

Table 2. Best-fit parameters for the X-ray spectra of 1ES 1210-646 from the observations described in this paper.

\begin{tabular}{|c|c|c|c|c|}
\hline $\begin{array}{c}\text { Model } \\
\text { parameter } \\
\end{array}$ & $\begin{array}{c}\text { XRT \#1 } \\
(0.3-9 \mathrm{keV})\end{array}$ & $\begin{array}{c}\text { XRT \#2 } \\
(0.3-9 \mathrm{keV})\end{array}$ & $\begin{array}{c}\text { XRT \#3 } \\
(0.3-9 \mathrm{keV}) \\
\end{array}$ & $\begin{array}{c}\text { JEM-X+IBIS } \\
(3-100 \mathrm{keV})\end{array}$ \\
\hline \multicolumn{5}{|l|}{ powerlaw $(+F e$ line $):$} \\
\hline$\chi^{2} /$ d.o.f. & 207.3/182 & $69.8 / 51$ & 188.6/147 & - \\
\hline$N_{\mathrm{H}}\left(\times 10^{21} \mathrm{~cm}^{-2}\right)$ & $5.2 \pm 0.4$ & $5.0_{-07}^{+0.8}$ & $5.1 \pm 0.5$ & - \\
\hline$\Gamma$ & $1.80 \pm 0.07$ & $1.63_{-0.14}^{+0.15}$ & $1.45 \pm 0.08$ & - \\
\hline Normalisation* & $11.5_{-10}^{+0.9}$ & $0.47^{-0.09}$ & $33 \pm 3$ & - \\
\hline$E(\mathrm{keV})$ & $6.71_{-0.03}^{+0.05}$ & $\underline{-0.01}$ & - & - \\
\hline$\sigma(\mathrm{keV})$ & $0.16_{-0.04}^{+0.05}$ & - & - & - \\
\hline$I\left(\times 10^{-4} \mathrm{ph} \mathrm{cm}^{-2} \mathrm{~s}^{-1}\right)$ & $5.8_{-12}^{+0.34}$ & - & - & \\
\hline \multicolumn{5}{|l|}{ bremsstrahlung $(+\mathrm{Fe}$ line $)$ : } \\
\hline$\chi^{2} /$ d.o.f. & $221.7 / 182$ & $72.3 / 51$ & $186.3 / 147$ & - \\
\hline$N_{\mathrm{H}}\left(\times 10^{21} \mathrm{~cm}^{-2}\right)$ & $4.3 \pm 0.3$ & $4.3_{-06}^{+0.7}$ & $4.7 \pm 0.4$ & - \\
\hline$k T_{\text {br }}(\mathrm{keV})$ & $7.3_{-11}^{+1.4}$ & $12_{-4}^{+9.6}$ & $22_{-6}^{+11}$ & - \\
\hline Normalisation* & $12.1_{-0 .}^{+0.6}$ & $0.56 \pm 0.04$ & $48 \pm 2$ & - \\
\hline$E(\mathrm{keV})$ & $6.72 \pm 0.04$ & - & - & - \\
\hline$\sigma(\mathrm{keV})$ & $0.17^{+0.06}$ & - & - & - \\
\hline$I\left(\times 10^{-4} \mathrm{ph} \mathrm{cm}^{-2} \mathrm{~s}^{-1}\right)$ & $6.2_{-1.3}^{+0.04}$ & - & - & \\
\hline \multicolumn{5}{|l|}{$B B+$ powerlaw: } \\
\hline$\chi^{2} /$ d.o.f. & - & - & - & $99.7 / 86$ \\
\hline$N_{\mathrm{H}}\left(\times 10^{21} \mathrm{~cm}^{-2}\right)$ & - & - & - & {$[5.0]$} \\
\hline$k T_{\mathrm{BB}}(\mathrm{keV})$ & - & - & - & $1.54_{-0.08}^{+0.06}$ \\
\hline$R_{\mathrm{BB}}^{\dagger}(\mathrm{km})$ & - & - & - & $5.9_{-1.9}^{+1.08}$ \\
\hline$\Gamma$ & - & - & - & $2.1 \pm 0.7$ \\
\hline$N^{*}$ pow & - & - & 一 & $2.9_{-2.8}^{+46.7}$ \\
\hline$C_{\text {calib }}$ & - & - & - & $2.0_{-17}^{+3.5}$ \\
\hline \multicolumn{5}{|l|}{ bremsstrahlung + powerlaw: } \\
\hline$\chi^{2} /$ d.o.f. & - & - & - & $120.4 / 86$ \\
\hline$N_{\mathrm{H}}\left(\times 10^{21} \mathrm{~cm}^{-2}\right)$ & - & - & - & {$[5.0]$} \\
\hline$k T_{\mathrm{br}}(\mathrm{keV})$ & - & - & - & $8.7_{-1.7}^{+2.7}$ \\
\hline$N^{*}$ br & - & - & - & $72_{-25}^{+10}$ \\
\hline$\Gamma$ & - & - & - & $1.5_{-4.3}^{0.7}$ \\
\hline$N^{*}$ pow & - & - & - & $1.7_{-17}^{+32.1}$ \\
\hline$C_{\text {calib }}$ & - & - & - & $0.13_{-0.06}^{+0.10}$ \\
\hline \multicolumn{5}{|l|}{ X-ray luminosity } \\
\hline $0.5-2 \mathrm{keV}$ & 0.2 & 0.1 & 0.7 & - \\
\hline $2-10 \mathrm{keV}$ & 0.4 & 0.2 & 1.9 & 2.0 \\
\hline $20-100 \mathrm{keV}$ & - & - & - & 0.1 \\
\hline
\end{tabular}

Notes. The above luminosities, corrected for interstellar Galactic absorption, are computed assuming a distance $d=2.8 \mathrm{kpc}$ (Masetti et al. 2009) and are expressed in units of $10^{35} \mathrm{erg} \mathrm{s}^{-1}$. In the cases in which the X-ray data could not completely cover the X-ray interval of interest for the luminosity determination, an extrapolation of the best-fit model is applied. ${ }^{(*)}$ In units of $10^{-3}$. $^{(\dagger)}$ Computed assuming a distance $d=2.8 \mathrm{kpc}$ to the source. ${ }^{(\ddagger)}$ Computed using the powerlaw model for the XRT spectra and the BB+powerlaw model for the JEM-X+IBIS spectrum.

may produce different normalisations for the spectra acquired by the two instruments.

Given that our past experience tells us that the intercalibration constant between these two instruments is $\sim 1$, we suggest that different values for this constant are likely to be due to the flux variability mentioned above. In any case, given that $1 \mathrm{ES}$ 1210-646 is more likely to be detected by IBIS during its high state, we consider that the JEM-X and IBIS spectra are representative of the same (high-intensity) state for the source and that it is therefore meaningful to combine them.

In the fits we fixed the neutral hydrogen column density to $5 \times 10^{21}$ atoms $\mathrm{m}^{-2}$, given that the lack of spectral coverage below $3 \mathrm{keV}$ (that is, the spectral range which is most sensitive to the $N_{\mathrm{H}}$ absorption effects) does not allow us to determine this parameter through the spectral fits.

The first remarkable issue of the JEM-X+IBIS spectral analysis is that no simple spectral model is able to fit the whole spectrum simultaneously, as none of them produces fits with reduced $\chi^{2}<2$. We thus tried combinations of two models;
Table 3. Values and upper limits to the EW of the iron emission line in 1ES 1210-646.

\begin{tabular}{lr}
\hline \hline Observation & $E W(\mathrm{keV})$ \\
\hline XRT \#1 & $1.6 \pm 0.3$ \\
XRT \#2 & $<0.9$ \\
XRT \#3 & $<0.2$ \\
JEM-X & $<0.4$ \\
\hline
\end{tabular}

Notes. A line width $(\sigma)$ of $0.16 \mathrm{keV}$ (see Table 2) was assumed to evaluate the above upper limits.

the best descriptions are obtained with $\mathrm{BB}+$ powerlaw and bremsstrahlung+powerlaw models (see Table 2).

Moreover, no iron emission line is apparent in the combined spectrum, with an upper limit on the EW as reported in Table 3. This is actually not surprising, given that the source emission level during which the considered JEM-X spectrum 

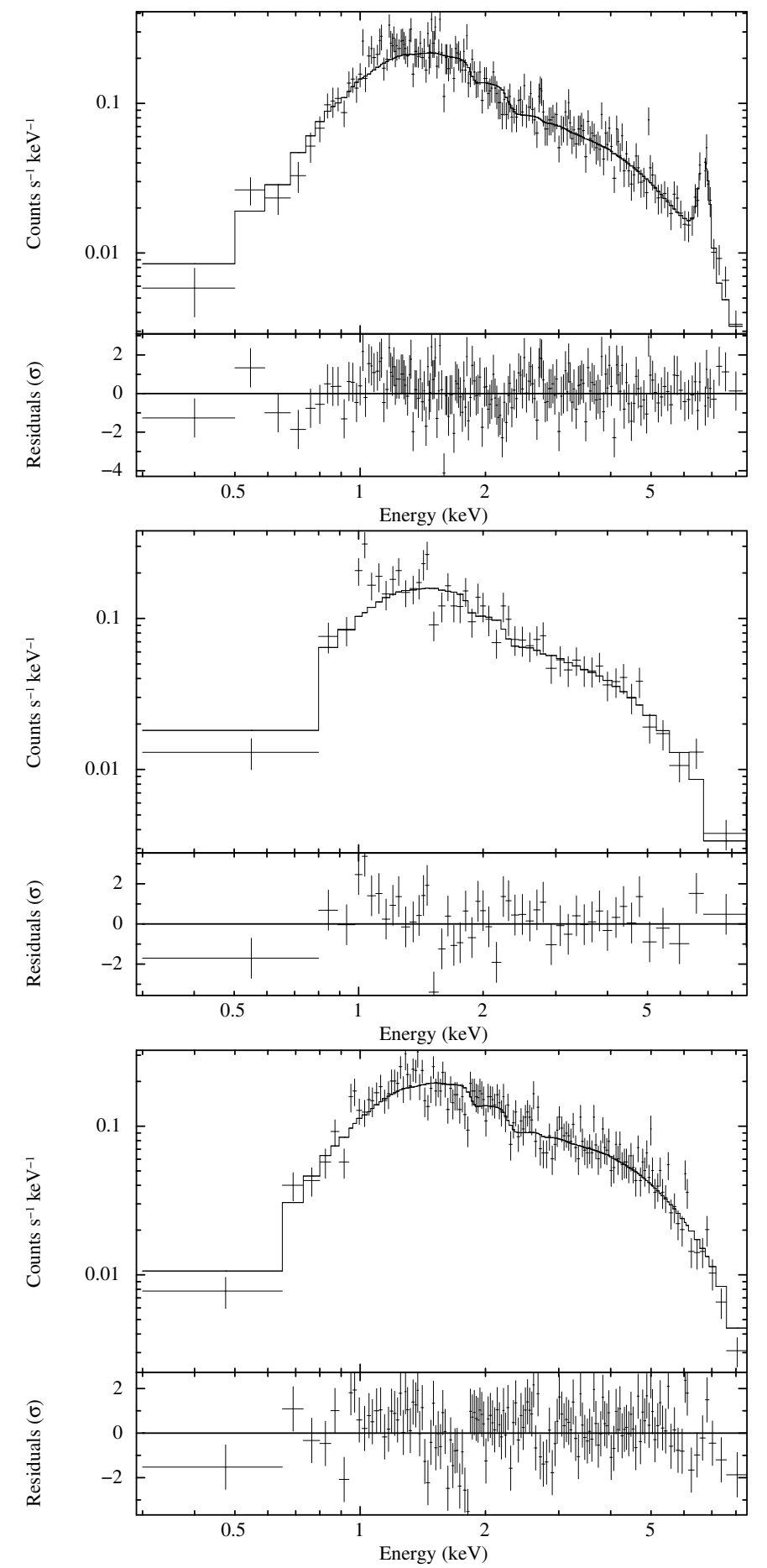

Fig. 3. X-ray spectra of 1ES 1210-646 acquired during XRT observations \#1 (upper panel), \#2 (central panel) and \#3 (lower panel), together with one of the best-fit models (an absorbed powerlaw in all cases; see Table 2 for details) and the corresponding fit residuals.

was accumulated was similar to the one of XRT observation \#3, in which no iron emission line was detected either.

Concerning instead the JEM-X observations for which no spectral information could be obtained, we found that Unit 1 of JEM-X detected the source at a flux of $4.7 \times 10^{-11} \mathrm{erg} \mathrm{cm}^{-2} \mathrm{~s}^{-1}$ in the 3-10 keV band, while Unit 2 could only provide a loose upper limit of $5.6 \times 10^{-11} \mathrm{erg} \mathrm{cm}^{-2} \mathrm{~s}^{-1}$ to the source flux, again in the $3-10 \mathrm{keV}$ band.

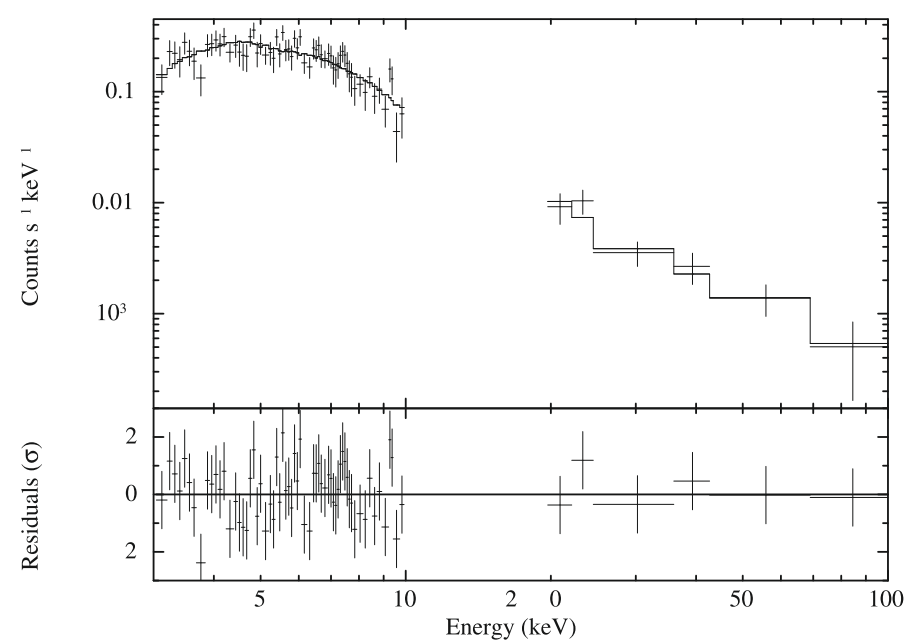

Fig. 4. Combined averaged JEM-X+IBIS X-ray spectrum of $1 E S$ 1210-646, plotted together with one of the best-fit models (absorbed BB plus powerlaw; see Table 2 for details) and the corresponding fit residuals.

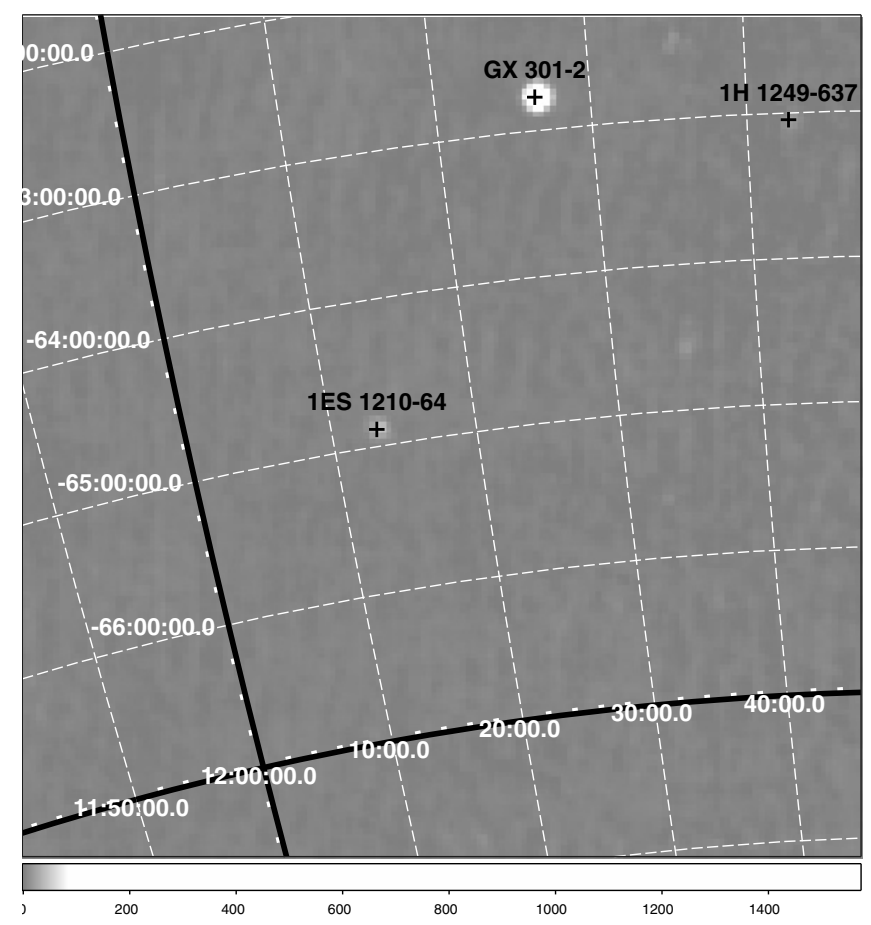

Fig. 5. WFCs mosaic image in the 3-17 keV band of the 1ES 1210-646 sky region.

\subsubsection{WFC data}

As reported Sect. 2.3, no spectrum of 1ES 1210-646 could be extracted from the WFC data. The source was clearly detected however, as Fig. 5 shows, in the mosaic maps of the summed WFCs data (for details on the mosaic production see Capitanio et al. 2008). The source is present in both the $3-17 \mathrm{keV}$ and the 17-28 keV mosaics maps at 50 and $20 \sigma$ confidence levels, respectively. 1ES 1210-646 is detected by the WFC at a flux of $\sim 2 \mathrm{mCrab}$ in the $3-28 \mathrm{keV}$ band, corresponding to $\sim 5.5 \times$ $10^{-11} \mathrm{erg} \mathrm{cm}^{-2} \mathrm{~s}^{-1}$; this value is compatible with the low-state JEM-X and the XRT pointing \#1 detections. Outside the period in which it was detected, following Verrecchia et al. (2007) we can assume a 3-28 keV detection limit of $<3 \times 10^{-11} \mathrm{erg} \mathrm{cm}^{-2} \mathrm{~s}^{-1}$ for 1ES 1210-646. 


\section{Discussion}

We here performed and presented, to the best of our knowledge, the first detailed spectral analysis of the X-ray emission from the X-ray binary 1ES 1210-646. We found that the source shows marked variability (of a factor of at least 10) on several timescales, from hundreds of seconds to months. Moreover, a strong transient iron emission line at $6.7 \mathrm{keV}$ is detected at intermediate X-ray fluxes only, and disappears when 1ES 1210-646 is at low and high intensity levels. Below we will discuss the characteristics of this X-ray system, the nature of its accretor, and the origin of its large and variable iron emission line in the light of the results shown in this paper.

\subsection{ES 1210-646: a peculiar HMXB}

Our results indicate that the $0.3-9 \mathrm{keV}$ X-ray spectrum of 1ES 1210-646 is described with a simple powerlaw, or a bremsstrahlung, while the INTEGRAL broadband data $(3-100 \mathrm{keV})$ are best fit with a combination of BB plus powerlaw or bremsstrahlung plus powerlaw (see Table 2). Because of the relatively low $\mathrm{S} / \mathrm{N}$ of the data we prefer the powerlaw (and the $\mathrm{BB}+$ powerlaw) description because i) the latter generally shows lower reduced $\chi^{2}$ values and ii) the JEM-X/IBIS intercalibration constant for the bremsstrahlung+powerlaw model is quite small $(\sim 0.1)$.

All the considered spectral models appear to be absorbed by the neutral interstellar hydrogen along the source line of sight only, indicating that the accreting material is likely not dense and/or neutral enough to contribute to the measured absorption acting on the X-ray emitted by the source.

As recalled in Sect. 1, Revnivtsev et al. (2007) put forward the identification of this X-ray source as a CV due to the presence of a large iron emission line at $6.7 \mathrm{keV}$ in the X-ray spectrum. However, as already remarked in Masetti et al. (2009), a number of HMXBs also show iron emission lines at this energy. Besides, the very large EW of the line $(\sim 1.6 \mathrm{keV}$, possibly due to the blend of iron lines, combined with the relatively low XRT spectral resolution) and its strong variability disfavour the CV interpretation for 1ES 1210-646 (for a comparison, see Landi et al. 2009, for a study of a sample of hard X-ray emitting CVs using XRT and IBIS). Also the high-state X-ray luminosity of 1ES 1210-646 is $\sim 1-2$ orders of magnitude larger than those typical of X-ray emitting CVs (see e.g. Barlow et al. 2006; Revnivtsev et al. 2008; Brunschweiger et al. 2009). Therefore we confidently rule out a CV interpretation for this system.

The possibility (see Masetti et al. 2009) that 1ES 1210-646 might be similar to the peculiar transient X-ray binary CI Cam (=XTE J0421+560), thought to host a white dwarf (e.g., Orlandini et al. 2000) can be ruled out as well: the X-ray variability of the latter source and its optical spectrum (see Orlandini et al. 2000, and references therein) are indeed markedly different with respect to those of 1ES 1210-646. Moreover, the presence of a $\mathrm{BB}$ emission with a temperature of $\sim 1.5 \mathrm{keV}$ in the X-ray spectrum of 1ES 1210-646 when the source shows high flux levels suggests that the accretor is more compact than a white dwarf.

We are also inclined to rule out a LMXB nature because these systems have quite different optical (and, to a lesser extent, $\mathrm{X}$-ray) spectra with respect to that of 1ES 1210-646. The huge variability of the iron emission line found in 1ES 1210-646 is never seen, as far as we know, in persistent LMXBs.

Besides, the length of the orbital period also disfavours the LMXB interpretation. While searching in the LMXB catalogues of Liu et al. (2007) and Ritter \& Kolb (2003), we found that very few systems have orbital periods of the order of days; and those that do actually show very different emission properties with respect to 1ES 1210-646. For instance, V395 Car (=2S 0921-630) with a period of $9.0 \mathrm{~d}$ (Branduardi-Raymont et al. 1983) has optical (e.g., Shahbaz et al. 1999) and X-ray (e.g., Kallman et al. 2003) spectra quite at variance with those of 1ES 1210-646. The same holds for Cyg X-2 (see Elebert et al. 2009; Di Salvo et al. 2002, and references therein), which has a period of $9.8 \mathrm{~d}$ (Casares et al. 1998). Likewise, LMXBs V404 Cyg (=GS 2023+338), with an orbital period of $6.4 \mathrm{~d}$ (Casares et al. 1992), is completely different from 1ES 1210-646 in the sense that the latter is a persistent although variable source, while the former is one of the best-known X-ray transient LMXBs hosting a dynamically-confirmed BH (cf. Kitamoto et al. 1989; Casares \& Charles 1994).

The binary 1ES 1210-646 might actually be more similar to the fast HMXB transient SAX J1819.3-2525, at least in terms of optical spectroscopic characteristics (Orosz et al. 2001; Maitra \& Bailyn 2006); however this system also displays a transient X-ray behaviour which is not seen in 1ES 1210-646 and moreover it too hosts a dinamically-confirmed BH (Orosz et al. 2001). Possibly 1ES 1210-646 will eventually evolve into a LMXB as Cyg X-2, as suggested for the HMXB Cir X-1 by Tauris \& Savonije (1999) and by Podsiadlowski et al. (2002).

All things considered, the HMXB interpretation for 1ES 1210-646 is the one best suited to explain the multiwavelength properties of this source. Nevertheless, the optical and X-ray spectra of 1ES 1210-646 are also anomalous for a persistent (albeit variable) HMXB. Indeed, the X-ray spectrum is atypical for an HMXB in the sense that we do not detect any break in its powerlaw shape below $10 \mathrm{keV}$ and below $100 \mathrm{keV}$ in the XRT and IBIS spectra, respectively, whereas HMXBs generally show spectral breaks in the 5-20 keV range (e.g., White et al. 1983). Actually, Corbet \& Mukai (2008) found a break around $6 \mathrm{keV}$ in the source X-ray spectrum: if our different description can be explained for XRT data as due to their narrower spectral coverage with respect to that of Corbet \& Mukai (2008), the INTEGRAL spectrum is possibly representative of a spectral state which is different from that reported by the above authors.

\subsection{The nature of the accretor}

The analysis presented here also indicates that a BB emission appears in the X-ray spectrum of 1ES 1210-646 when the source is at its high state. This latter fact is however not surprising because according to Hickox et al. (2004) soft excesses are quite ubiquitous in HMXB spectra. The BB component detected in the X-ray spectrum of 1ES 1210-646 is consistent with an emission from an accreting NS surface, both in terms of the emitting area and temperature. This agrees with the conclusions of Hickox et al. (2004), according to which a soft excess in HMXBs with X-ray luminosities of less than $10^{36} \mathrm{erg} \mathrm{s}^{-1}$ can be produced by thermal emission from the surface of the accreting NS (as in the case of the low-luminosity HMXB X Per; Coburn et al. 2001). That we see this $\mathrm{BB}$ component only at the highest emission levels for this source is likely a selection effect in the sense that it is more apparent when the NS accretion rate is highest, whereas during lower flux levels the non-thermal (i.e., powerlaw) component dominates.

Moreover, as we detect a BB component with an emitting area comparable to the surface of a NS (or at least a large fraction of it; see Table 2) in 1ES 1210-646, the accreting object is probably an NS with a relatively low magnetic field; in this case, 
no accretion column would form, and the detection of pulsed $\mathrm{X}$-ray emission would be rather difficult. Consequently the accretor in 1ES 1210-646 would be an analogue of the one in the HMXB Cir X-1 (see Jonker et al. 2007, and references therein), which is believed to be a low magnetic field NS.

It is also worth pointing out that this source is interesting from a further point of view, as it seems to be one of the very rare $\mathrm{Be} / \mathrm{X}$-ray binaries in the Galaxy with spectral type beyond B2 (Negueruela 1998). Actually, according to the Corbet diagram (Corbet 1986), a 6.7 day orbital period is quite short for a $\mathrm{Be} / \mathrm{X}$-ray binary and would be better suited to a supergiant system (which is ruled out by Masetti et al. 2009), unless the hosted NS is rapidly spinning $\left(P_{\text {spin }} \lesssim 10^{-2} \mathrm{~s}\right)$. Otherwise, the NS could be rotating substantially slower, with $P_{\text {spin }} \sim 10^{2}$ s, and accretes from the stellar wind of the companion star.

\subsection{Origin of the iron emission line}

The possible origin of the $6.7 \mathrm{keV}$ emission line generally observed in the X-ray binaries is the $\mathrm{K}_{\alpha}$ transition of highly ionised He-like iron. This can be produced either in a thin hot plasma region with an electron temperature of several $\mathrm{keV}$ (as usually observed in disc-accreting X-ray binaries; cf. White et al. 1995), or by radiative recombination followed by electron cascade transition in a photoionised plasma with a relatively low temperature (see Liedahl \& Paerels 1996, and references therein).

The latter mechanism is at work in HMXBs like SMC X-1 (Vrtilek et al. 2001), Vela X-1 (Goldstein et al. 2004) and 4U 1700-37 (van der Meer et al. 2005): it makes the emission lines more easily detectable during eclipses, when the X-ray beam irradiating the stellar wind is not directly visible but the reprocessed emission from the photoionised stellar wind is. However, this effect does not explain the iron line variability that we detect in 1ES 1210-646, as in this case the line is apparent far from the possible eclipses of the system (see below), assuming the ephemeris of Corbet \& Mukai (2008).

The X-ray binary Cyg X-3 also shows an iron emission line at $6.7 \mathrm{keV}$, likely emitted from the accreted stellar wind surrounding the accretor and strongly photoionised by its highenergy radiation (see e.g. Szostek et al. 2008, and references therein); but in this object the intensity of the $6.7 \mathrm{keV}$ iron line as a function of the source flux (Hjalmarsdotter et al. 2009) reaches its lowest levels when Cyg X-3 is at intermediate flux levels, thus at variance with respect to the behaviour shown by $1 \mathrm{ES}$ 1210-646.

This accordingly suggests that the iron emission detected in this latter source is produced in a highly ionised, hot and thin accretion stream rather than by a relatively cold photoionised plasma.

In addition, the line variability behaviour of 1ES 1210-646 is reminiscent of that displayed by GX 301-2, an HMXB showing an iron emission at $6.4 \mathrm{keV}$ with $\mathrm{EW}$ varying by a factor of $\sim 6$ depending on the orbital phase of the NS (Nagase 1989; Endo et al. 2002). In particular, in this source the iron emission line is stronger when the accreting NS is close to the periastron (Endo et al. 2002). Can this effect be present in 1ES 1210-646 as well? Indeed, according to the orbital ephemeris of Corbet \& Mukai (2008), we find that XRT observation \#1 falls at orbital phase $\phi \sim 0$ (when the orbital modulation of the source flux reaches its maximum, that is, at or near the periastron), while the other XRT pointings and our JEM-X spectrum were acquired at orbital phases of $\sim 0.3, \sim 0.8$ and $\sim 0.3$, respectively. Likewise,
Corbet \& Mukai (2008) also find an iron emission in an X-ray spectrum acquired near an orbital phase of 0 .

We note however that the line energy in GX 301-2 indicates that the emission in this source is due to fluorescence of neutral iron irradiated by the accreting NS, whereas (as already remarked) in 1ES 1210-646 the line at $6.7 \mathrm{keV}$ comes from a highly ionised medium. Nevertheless, the line intensity behaviour in these two sources as a function of the orbital phase is apparently very similar.

Therefore, the presence of a variable emission line in 1ES 1210-646 as a function of its orbital period with a detectable intensity as the accretor approaches periastron suggests that this feature is tied to the increase of the accretion rate from the secondary star (likely from its wind) onto the NS due to its orbital motion. The line thus possibly forms in a small, transient and highly ionised accretion figure around the NS itself.

\section{Conclusions}

From our X-ray data analysis carried out with several instruments on board of different spacecraft, we conclude that the best interpretation for the nature of the X-ray source 1ES 1210-646 is that it is an HMXB hosting a low magnetic field NS, although it is a system with several peculiarities like its optical and X-ray spectra and the large variability of the iron emission line at $\sim 6.7 \mathrm{keV}$. This variable line is apparently produced in a highly ionised and transient accretion figure around the accreting NS during the periastron passage. In order to further explore the peculiar nature of 1ES 1210-646 and its behaviour as a function of the orbital phase, higher S/N optical and X-ray spectra of the source along with multiwavelength monitoring and a deeper timing analysis to search for short-term modulations (from milliseconds to hours), are definitely needed.

Acknowledgements. We thank Domitilla de Martino and Margaretha Pretorius for preliminar comments and discussions on the nature of this source, Mauro Orlandini for discussions on HMXB systems and X-ray data analysis, and Vanessa McBride for comments and suggestions. We also thank the anonymous referee for useful remarks which helped us to improve the paper. This research has made use of the ASI Science Data Center Multimission Archive, of the WFC archive at INAF/IASF di Roma, of the SIMBAD database operated at CDS, Strasbourg, France, and of the NASA Astrophysics Data System Abstract Service. Some of the authors acknowledge the ASI and INAF financial support via grant No. I/008/07.

\section{References}

Anders, E., \& Grevesse, N. 1989, Geochim. Cosmochim. Acta, 53, 197

Barlow, E. J., Knigge, C., Bird, A. J., et al. 2006, MNRAS, 372, 224

Bird, A. J., Barlow, E. J., Bassani, L., et al. 2006, ApJ, 636, 765

Bird, A. J., Malizia, A., Bazzano, A., et al. 2007, ApJS, 170, 175

Boella, G., Butler R. C., Perola, C., et al. 1997, A\&AS, 122, 299

Blackburn, J. K. 1995, FTOOLS: A FITS Data Processing and Analysis Software

Package, in Astronomical Data Analysis Software and Systems IV, ed. R. A. Shaw, H. E. Payne, \& J. J. E. Hayes, ASP Conf. Ser., 77, 367

Branduardi-Raymont, G., Corbet, R. H. D., Mason, K. O., et al. 1983, MNRAS, 205, 403

Brunschweiger, J., Greiner, J., Ajello, M., \& Osborne, J. 2009, A\&A, 496, 121 Burrows, D. N., Hill, J. E., Nousek, J. A., et al. 2005, Space Sci. Rev., 120, 165 Capitanio, F., Bird, A. J., Federici, M., Bazzano, A., \& Ubertini, P. 2008, Correlating the WFC and the IBIS hard X-ray surveys, in A Population Explosion: The Nature \& Evolution of X-ray Binaries in Diverse Environments, ed. R. M. Bandyopadhyay, S. Wachter, D. Gelino, \& C. R. Gelino, AIP Conf. Proc., 1010, 257

Casares, J., \& Charles, P. A. 1994, MNRAS, 271, L5

Casares, J., Charles, P. A., \& Naylor, T. 1992, Nature, 355, 614 Casares, J., Charles, P. A., \& Kuulkers, E. 1998, ApJ, 493, L39 Coburn, W., Heindl, W. A., Gruber, D. E., et al. 2001, ApJ, 552, 738 Corbet, R. H. D. 1986, MNRAS, 220, 1047 
Corbet, R. H. D., \& Mukai, K. 2008, ATel, 1861

Cusumano, G., La Parola, V., Segreto, A., et al. 2009, A\&A, 510, A48

Di Salvo, T., Farinelli, R., Burderi, L., et al. 2002, A\&A, 386, 535

Dorman, B., \& Arnaud, K. A. 2001, Redesign and reimplementation of XSPEC in Astronomical Data Analysis Software and Systems X, ed. F. R. Harnden Jr., F. A. Primini, \& H. E. Payne (San Francisco: ASP), ASP Conf. Ser., 238, 415

Elebert, P., Callanan, P. J., Torres, M. A. P., \& Garcia, M. R. 2009, MNRAS, 395, 2029

Elvis, M., Plummer, D., Schachter, J., \& Fabbiano, G. 1992, ApJS, 80, 257

Endo, T., Ishida, M., Kuniaki, M., et al. 2002, ApJ, 574, 879

Forman, W., Jones, C., Cominsky, L., et al. 1978, ApJS, 38, 357

Gehrels, N., Chincarini, G., Giommi, P., et al. 2004, ApJ, 611, 1005

Goldstein, G., Huenemoerder, D. P., \& Blank, D. 2004, AJ, 127, 2310

Hickox, R. C., Narayan, R., \& Kallman, T. R. 2004, ApJ, 614, 881

Hjalsmarsdotter, L., Zdziarski, A. A., Szostek, A., \& Hannikainen, D. 2009, MNRAS, 392, 251

Jager, R., Mels, W. A., Brinkman, A. C., et al. 1997, A\&AS, 125, 557

Jonker, P. G., Nelemans, G., \& Bassa, C. G. 2007, MNRAS, 374, 999

Kallman, T. R., Angelini, L., Boroson, B., \& Cottam, J. 2003, ApJ, 583, 861

Kitamoto, S., Tsunemi, H., Miyamoto, S., et al. 1989, Nature, 342, 518

Krivonos, R., Revnivtsev, M., Lutovinov, A., et al. 2007, A\&A, 475, 775

Landi, R., Bassani, L., Dean, A. J., et al. 2009, MNRAS, 392, 630

Lebrun, F., Leray, J. P., Lavocat, P., et al. 2003, A\&A, 411, L141

Liedahl, D. A., \& Paerels, F. 1996, ApJ, 468, L33

Liu, Q. Z., van Paradijs, J., \& van den Heuvel, E. P. J. 2007, A\&A, 469, 807

Lund, N., Budtz-Jørgensen, C., Westergaard, N. J., et al. 2003, A\&A, 411, L231

Maitra, D., \& Bailyn, C. D. 2006, ApJ, 637, 992

Masetti, N., Parisi, P., Palazzi, E., et al. 2009, A\&A, 495, 121

Mewe, R., Groenschild, E. H. B. M., \& van den Oord, G. H. J. 1985, A\&AS, 62, 197

Morrison, R., \& McCammon, D. 1983, ApJ, 270, 119
Nagase, F. 1989, PASJ, 41, 1

Negueruela, I. 1998, A\&A, 338, 505

Orlandini, M., Parmar, A. N., Frontera, F., et al. 2000, A\&A, 356, 163

Orosz, J. A., Kuulkers, E., van der Klis, M., et al. 2001, ApJ, 555, 489

Podsiadlowski, P., Rappaport, S., \& Pfahl E. D. 2002, ApJ, 565, 1107

Predehl, P., \& Schmitt, J. H. M. M. 1995, A\&A, 293, 889

Press, W. H., Teukolsky, S. A., Vetterling, W. T., \& Flannery, B. P. 1992, Numerical Recipes (Cambridge: Cambridge Univ. Press)

Protassov, R., Van Dyk, D. A., Connors, A., Kashyap, V. L., \& Siemiginowska, A. 2002, ApJ, 571, 545

Revnivtsev, M., Sunyaev, R., Lutovinov, A., \& Sazonov, S. 2007, ATel, 1253

Revnivtsev, M., Sazonov, S., Krivonos, R., Ritter, H., \& Sunyaev, R. 2008, A\&A, 489, 1121

Reynolds, A. P., Parmar, A. N., Hakala, P. J., et al. 1999, A\&AS, 134, 287

Ritter, H., \& Kolb, U. 2003, A\&A, 404, 301

Romano, P., Campana, S., Chincarini, G., et al. 2006, A\&A, 456, 917

Schlegel, D. J., Finkbeiner, D. P., \& Davis, M. 1998, ApJ, 500, 525

Shahbaz, T., Kuulkers, E., Charles, P. A., et al. 1999, A\&A, 344, 101

Szostek, A., Zdziarski, A. A., \& McCollough, M. L. 2008, MNRAS, 388, 1001

Tauris T. M., \& Savonije, G. J. 1999, A\&A, 350, 928

Tueller, J., Baumgartner, W. H., Markwardt, C. B., et al. 2010, ApJS, 186, 378

Ubertini, P., Lebrun, F., Di Cocco, G., et al. 2003, A\&A, 411, L131

van der Meer, A., Kaper, L., Di Salvo, T., et al. 2005, A\&A, 432, 999

Verrecchia, F., in 't Zand, J. J. M., Giommi, P., et al. 2007, A\&A, 472, 705

Vrtilek, S. D., Raymond, J. C., Boroson, B., et al. 2001, ApJ, 563, L139

Westergaard, N. J., Kretschmar, P., Oxborrow, C. A., et al. 2003, A\&A, 411, L257

White, N. E., Swank, J. H., \& Holt, S. S. 1983, ApJ, 270, 711

White, N. E., Nagase, F., \& Parmar, A. N. 1995, The properties of X-ray binaries, in X-ray binaries, ed. W. H. G. Lewin, J. van Paradijs, \& E. P. J. van den Heuvel (Cambridge: Cambridge Univ. Press), 1

Winkler, C., Courvoisier, T. J.-L., Di Cocco, G., et al. 2003, A\&A, 411, L1 